\title{
A Novel Model of the Atom
}

\author{
Hans W. Giertz \\ Giertz Tech, Gnesta, Sweden \\ Email: hans@miklagaard.com
}

Received 22 January 2016; accepted 26 February 2016; published 29 February 2016

Copyright (C) 2016 by author and Scientific Research Publishing Inc. This work is licensed under the Creative Commons Attribution International License (CC BY). http://creativecommons.org/licenses/by/4.0/

(c) (i) Open Access

\begin{abstract}
In this study all energy in the universe, here called energy quanta, originate from a singularity at the centre of the universe. These energy quanta have different frequencies and at each frequency the energy quanta can have positive or negative spin direction. There is a force of attraction between energy quanta which have exactly the same frequency but opposite spins. This is the dominating force in the universe and accounts for the strong nuclear force, the Coulomb force and the gravitational force. The universe contains one more basic entity; the oscillator quantum which absorbs and re-emits energy quanta at one specific frequency. The oscillator quantum can have positive or negative spin. Thus, there is a force of attraction between oscillator quanta with opposite spins and which amalgamates oscillator quanta into larger structures, i.e. particles (e.g. electron). These particles also have spin at a specific spin frequency and they have positive or negative spin. Thus they absorb and re-emit energy quanta at a frequency specific to the particle and where they can have positive or negative spin. This amalgamates particles into larger structures, e.g. quarks, neutron, proton and atomic nucleus. Using this model enables simple and stringent descriptions of elementary particle physics, electromagnetic theory, gravity, photon and inertial mass. The present model may be a step towards unification of elementary particle physics, general relativity, quantum physics and electromagnetic theory into one comprehensive theory.
\end{abstract}

\section{Keywords}

Electron, Atom, Gravity, Photon, Mass, Strong Force, Standard Model, Theory of Everything

\section{Introduction}

Here a novel theoretical model of the atom is proposed. The model builds on a universe which is rather simple. The centre of the universe emits vast amount of energy consisting of energy quanta. An energy quantum has a specific frequency and spin direction which can be positive or negative spin. Forces in the universe originate from these energy quanta. There is a repelling force between energy quanta which have the same frequency and 
spin direction. There is force of attraction between energy quanta which have the same frequency and opposite spin direction. The centre of the universe emits a broad spectrum of energy quanta with different frequencies and positive as well as negative spin.

Furthermore, the novel model builds on the proposal that the universe consists of only two basic entities; the energy quantum and the oscillator quantum. The oscillator quantum has spin and its spin frequency and spin direction creates resonance with energy quanta which have the same spin frequency and spin direction; thus, it absorbs and re-emits energy quanta with the same frequency and spin direction. Hence, the oscillator quantum is a forced damped oscillator. All oscillator quanta in the universe have the same spin frequency; however positive or negative spin. Thus, there is force of attraction between oscillator quanta with opposite spin because there is force of attraction between re-emitted energy quanta. Clusters of oscillator quanta also have spin at a specific frequency and positive or negative spin direction; thus, they absorb and re-emit energy quanta with the same spin frequency and spin direction. Hence, a cluster of oscillator quanta is also a forced damped oscillator. There is repelling force between clusters where the re-emitted energy quanta have the same spin frequency and spin direction. There is force of attraction between clusters where the re-emitted energy quanta have the same spin frequency and opposite spin direction. It is proposed that the atom consist of different clusters of oscillator quanta. A cluster can constitute an electron, a positron, a quark, a neutron, a proton and the atomic kernel. Each cluster has its specific spin frequency; however, it can have positive or negative spin. This creates force of attraction between electron and positron, between quarks and between neutrons and protons. These forces amalgamate the atom. The exchange of energy quanta which have opposite spin directions are what is called the strong force and the gluon in the Standard Model [1].

Essential parts of this theory have already been published. However, three major changes are introduced which makes the theory simpler and more accurate. In this novel theory energy quanta have spin and there is a force between energy quanta which have the same frequency and opposite spin (attracting force) or the same spin (repelling force). In previous studies, [2] [3], it was assumed that energy quanta did not have spin and that there was force of attraction between energy quanta having slightly different frequencies [2]. In this novel model the universe contains only two basic entities; the energy quantum and the oscillator quantum. All oscillator quanta in the universe have the same frequency and they have positive or negative spin; hence, there is only one unique oscillator quantum and it can have positive or negative spin. Previous reports, [2] [3], assumed that there were a number of oscillator quanta with different frequencies and where each had specific properties. In this novel model different configurations of oscillator quanta provide unique properties like electron, positron, quarks, neutron and proton.

The above theory has been mapped previously on the electron [2] [3], although with the constraints mentioned above. These reports describe electromagnetic theory in a novel way entirely based on the electron's absorbed and re-emitted energy quanta. The electron charge $Q$ is the re-emitted energy quanta flow density at distance $r=$ 0 and the current $\boldsymbol{i}$ is the transport, with velocity $\boldsymbol{v}$, of this re-emitted energy quanta flow density. The displacement $\boldsymbol{D}$ (electric field) is the re-emitted energy quanta flow density at distance $r$ and the magnetic field $\boldsymbol{H}(r)$, from transported electrons, is the time derivate of observed re-emitted energy quanta flow density at distance $r$. This previously published electromagnetic theory was compared with traditional electromagnetism [4] [5]. It has been shown how energy quanta originating from the centre of the universe can be measured as well as the direction towards this source [3]. It has been shown that the gravitational force is the result of energy quanta exchanged between bodies [2]. It has been shown that the photon is enforced energy $h v$ super positioned on energy quanta [2] and which was compared with the state of the art model of the photon [6]. It has also been shown that inertial mass is the result of enforced energy $h v$ super positioned on re-emitted energy quanta [2]. However, in [2] gravity, photon and inertial mass were described with the constraints described above. Therefore, an enhanced description of gravity, photon and inertial mass is included in the present theoretical model. This novel description of inertial mass is here compared with today's description based on the Higgs boson and the Higgs field [7]-[11]. The present theoretical model is fundamentally different to the Standard Model. Here all energy originates from the centre of the universe and the atom is complete passive; it absorbs and re-emits energy quanta which results in stored energy, forces and fields. The Standard model, quantum physics [12], general relativity [13], electromagnetic theory [4] [5] and string theory [14] describes a different universe where energy, forces and fields are inherent properties of the electron and the atom, detached from external influence. A comparison with these disciplines is made. 


\section{Theoretical Model}

\subsection{General}

Today physics is described by different models: the Standard Model [1], quantum physics [12], general relativity [13], electromagnetic theory [4] [5] and string theory [14]. These theories have a common denominator. It is assumed that the characteristics of the electron and atomic nucleus, such as charge, fields, forces and energy, are intrinsic properties. Hence, today's physics claims that they are generated without external influence [1] [4] [5] [12]-[14]. The exception is the Higgs Field and the Higgs Boson [7]-[9].

This paper proposes that it is quite the opposite. The atom in itself contains no forces or energy, what so ever. The atom is completely passive. All energy in the universe originates from a singularity, positioned at the centre of the universe. This energy is absorbed by the electron and the atomic nucleus and the absorbed and re-emitted energy result in phenomena observed in physics: stored energy, forces, current and fields.

This Section is structured in the following way. It starts with a description of energy quanta. A model of the electron and the positron is presented, including a description of absorbed and re-emitted energy quanta. The same model is then utilized to explain the structure of the nucleus and the strong nuclear force. The strong nuclear force is created by a process similar to the Coulomb force, albeit by energy quanta and oscillator quanta with frequencies specific to the nucleus and the strong nuclear force.

The same model is once again utilized to explain gravity. Gravity is created by a process similar to the Coulomb force, albeit by energy quanta and oscillator quanta with frequency specific to gravity.

The same model is finally utilized to explain the photon and inertial mass.

\subsection{Singularity}

According to the theoretical model the universe contains a singularity. This singularity has been described previously [2] [3], although with the constraints described in Section 1 . This singularity generates energy quanta at different frequencies and they can have positive or negative spin. The energy quantum has no mass and propagates with the speed of light. An energy quantum is described by its vector $\delta \boldsymbol{S}_{\omega}$ in the present study, where $\omega$ denotes its frequency $\omega$. The energy quantum $\delta \boldsymbol{S}_{\omega}$ remains unaltered as it travels through space. The singularity emits energy quanta $\delta \boldsymbol{S}_{\omega}$ uniformly into space creating energy flow density $\boldsymbol{S}_{\omega}(0)$ at the singularity and energy flow density $\boldsymbol{S}_{\omega}(R)$ at the distance $R$. Energy quanta $\delta \boldsymbol{S}_{\omega}$ are radiated uniformly into space, which results in that $S_{\omega}(R)$ decreases with the area of the sphere

$$
\boldsymbol{S}_{\omega}(R)=\boldsymbol{S}_{\omega}(R=0) \frac{1}{4 \pi R^{2}} .
$$

The energy quanta can have positive and negative spin and which is denoted $\delta \boldsymbol{S}_{\omega}^{+}$respectively $\delta \boldsymbol{S}_{\omega}^{-}$.

\subsection{Oscillator Quantum}

It is proposed that the universe contains only two basic entities: energy quanta and one oscillator quantum. The oscillator quantum is a forced damped oscillator with natural frequency $\omega_{0}$. It absorbs energy quanta $\delta \boldsymbol{S}_{\omega o}$ and energy flow density $\boldsymbol{S}_{\omega o}(R)$, having the same frequency as the oscillator quantum natural frequency. However, the energy quanta have positive spin $\delta \boldsymbol{S}_{\omega o}^{+}$or negative spin $\delta \boldsymbol{S}_{\omega o}^{-}$which implies that the oscillator quantum also has positive or negative spin. An oscillator quantum absorbs only energy quanta having the same spin frequency and spin direction. The absorbed energy quanta cannot be stored in the oscillator quantum and are consequently re-emitted uniformly into space as energy flow density $\boldsymbol{S}_{\omega o}^{+}(r)$ respectively $\boldsymbol{S}_{\omega o}^{-}(r)$ at the distance $r$ from the oscillator quantum. Hence, the energy flow density re-emitted by the oscillator quantum is

$$
\boldsymbol{S}_{\omega o}(r)=\alpha \boldsymbol{S}_{\omega o}(R=0) \frac{1}{4 \pi R^{2}} \cdot \frac{1}{4 \pi r^{2}},
$$

where $\alpha$ is a constant which mirrors the oscillator quantum transfer function. However, at the earth the distance $R$ to the singularity is constant. Equation (2) can, thus, be simplified into

$$
\boldsymbol{S}_{\omega o}(r)=\boldsymbol{S}_{\omega o}(r=0) \frac{1}{4 \pi r^{2}} .
$$


From now on $\boldsymbol{S}_{\omega o}(r=0)$ is denoted $\boldsymbol{S}_{\omega o}(0)$. Equation (3) describes the energy flow density from one oscillator quantum. There is repelling force between two energy quanta which have the same frequency and spin direction and force of attraction between two energy quanta which have the same frequency but opposite spin directions. Now we picture an energy quantum, $\delta \boldsymbol{S}_{\omega o}$, with frequency $\omega_{o}$ and which passes close to an oscillator quantum with frequency $\omega_{0}$. The energy quantum imposes an impulse and force $\delta \boldsymbol{F}_{\omega o}$ on the oscillator quantum. This force is directed towards the propagating energy quantum when the energy quantum and the oscillator quantum have opposite spins. This force is directed along the propagating energy quantum when the energy quantum and the oscillator quantum have the same spin. The force $\delta \boldsymbol{F}_{\omega o}$ on the oscillator quantum, as a result of a passing energy quantum $\delta \boldsymbol{S}_{\omega o}$, is

$$
\delta \boldsymbol{F}_{\omega o}=\beta \delta \boldsymbol{S}_{\omega o},
$$

where $\beta$ is a constant. Energy flow density $\boldsymbol{S}_{\omega o}(r)$ re-emitted and propagating from another oscillator quantum, at the distance $r$, towards this oscillator quantum imposes a force $\boldsymbol{F}_{\omega o}(r)$ on the oscillator quantum

$$
\boldsymbol{F}_{\omega o}(r)=\eta \boldsymbol{S}_{\omega o}(r),
$$

where $\eta$ is a constant. According to Equation (3) this can be re-written into

$$
\boldsymbol{F}_{\omega o}(r)=\eta S_{\omega o}(0) \frac{\boldsymbol{r}}{4 \pi r^{3}} \text {. }
$$

The total force acting on $n$ oscillator quanta is

$$
\boldsymbol{F}_{\omega o}^{n}(r)=\eta n S_{\omega o}(0) \frac{\boldsymbol{r}}{4 \pi r^{3}} .
$$

The total energy flow density $\boldsymbol{S}_{\omega o}(r)$, re-emitted by $m$ oscillator quanta in a cluster, is $m \boldsymbol{S}_{\omega o}(r)$. Consequently, the force $\boldsymbol{F}_{\omega o}^{m n}(r)$ imposed from one cluster of $m$ oscillator quanta, acting on another cluster of $n$ oscillator quanta at distance $\boldsymbol{r}$, is

$$
\boldsymbol{F}_{\omega o}^{m n}(r)=\xi_{o} m n \frac{\boldsymbol{r}}{4 \pi r^{3}},
$$

where $\xi_{o}$ is a constant, valid for oscillator quanta with frequency $\omega_{o}$. Here $r$ is much larger than the distances within each cluster of oscillator quanta. Equations ((5)-(8)) result in repelling force when oscillator quanta in the two clusters have the same spin and force of attraction when they have opposite spins.

\subsection{Electron and Positron}

It is proposed that the electron consists of two oscillator quanta with opposite spin directions. This creates a strong force between the two oscillator quanta according to Equation (8) and which amalgamates them into a configuration. This configuration of two oscillator quanta also has spin at its spin frequency $\omega_{e}$, where $e$ denotes electron. Consequently, the configuration of two oscillator quanta is a forced damped oscillator with natural frequency $\omega_{e}$. The spin is negative, whereby it absorbs energy quanta and re-emits energy flow density $\boldsymbol{S}_{\omega e}^{-}(r)$ in the same way as the oscillator quantum, with the exception that the absorbed and re-emitted energy quanta have the frequency $\omega_{e}$. The re-emitted energy quanta flow density $S_{\omega e}^{-}(0)$ is the electron charge and the re-emitted energy quanta flow density $\boldsymbol{S}_{\omega e}^{-}(r)$ is the displacement $\boldsymbol{D}$. The transport of $\boldsymbol{S}_{\omega e}^{-}(0)$ with velocity $\boldsymbol{v}$ is the current and the observed time derivate of $\boldsymbol{S}_{\omega e}^{-}(r)$ at distance $r$, from travelling electrons, describes the magnetic field $\boldsymbol{H}(r)$. This has been described previously in detail [2] [3]; however with the constraints described in Section 1. Electrons have the same spin and consequently, they repel each other according to Equation (8). However, where the equation is valid at the electron frequency $\omega_{e}$ and for the electron constant $\xi_{e}$;

$\boldsymbol{F}_{\omega e}^{m n}(r)=\xi_{e} m n \frac{\boldsymbol{r}}{4 \pi r^{3}}$. This expresses the Coulomb force. Hence, there is no principle difference between the force created between oscillator quanta and the force created between electrons other than that they operate in two different frequency domains.

The positron is identical to the electron; however, it has positive spin and the absorbed energy quanta and the re-emitted energy flow density $\boldsymbol{S}_{\omega e}^{+}(r)$ have positive spin. Consequently, two positrons repel according to Eq- 
uation (8) because they have the same spin. There is force of attraction between a positron and an electron because they have the same spin frequency and opposite spin directions.

\subsection{Atom}

It is of course impossible to describe the atom in its exact details. The following is an attempt to describe a common principle which may explain layers of the atom. The description is schematic and simplified and the purpose is only to highlight principles.

It is proposed that the atom contains entities which are smaller than the quark; here they are called electron/positron and micro quark $(\mu q)$. The electron/positron is a configuration (called $e p$ ) which consists of one electron and one positron, amalgamated by the force of attraction according to Equation (8). This configuration has spin at the frequency $\omega_{e p}$ and the spin can be positive or negative. Thus, the configuration is a forced damped oscillator with natural frequency $\omega_{e p}$. The configuration absorbs and re-emits energy quanta with the frequency $\omega_{e p}$. Hence, there is force of attraction between electron/positron configurations which have opposite spin directions. A $\mu q$ can for instance consist of twelve electron/positron configurations oriented in a ring and where adjacent configurations have opposite spins, i.e. the ring consist of $e p^{+}, e p^{-}, e p^{+}, e p^{-}, e p^{+}$etc. Hence, there is a total force of attraction between adjacent electron/positron configurations in the ring according to Equation (8). This configuration has a spin in itself at its frequency $\omega_{\mu q}$ where $\mu q$ denotes micro quark. The configuration absorbs energy quanta and re-emits energy flow density $\boldsymbol{S}_{\omega \mu q}(r)$ in the same way as the oscillator quantum, the electron and the electron/positron configuration, with the exception that the absorbed and re-emitted energy quanta have the frequency $\omega_{\mu q}$. The $\mu q$ can have positive or negative spin. There are three different micro quarks and where the configuration of $e p$ are somewhat different (e.g. different number of electron/positron configurations in the ring) which result in that each type of $\mu q$ has its specific spin frequency, i.e. $\omega_{\mu q 1}, \omega_{\mu q 2}$ and $\omega_{\mu q 3}$ and where each can have positive or negative spin directions.

The configuration may resemble one of the most common structures in chemistry; the biphenyl ring where two rings of each six carbon atoms are connected forming a structure of twelve carbon atoms (and 10 hydrogen atoms) in total. By studying common structures in chemistry we may have an insight into the atom. Physics may be simple and repetitive; guarding those structures which exhibit maximum stability or minimum energy level.

There are three different quarks. The quarks may consist of rings, each consisting of twelve micro quarks $(\mu q)$ with the same spin frequency. One quark, here called quark type $1\left(q_{1}\right)$ may consist of a ring containing the same type of $\mu q$, e.g. $\mu q_{1}$. Adjacent $\mu q_{1}$ have opposite spin, i.e. the ring consist of $\mu q_{1}{ }^{+}, \mu q_{1}{ }^{-}, \mu q_{1}{ }^{+}, \mu q_{1}{ }^{-}, \mu q_{1}{ }^{+}$and $\mu q_{1}{ }^{-}$ etc. Hence, there is a total force of attraction between adjacent $\mu q$ in the ring according to Equation (8). This configuration has spin in itself at its natural frequency $\omega_{q}$, where $q$ denotes quark. The configuration absorbs energy quanta and re-emits energy flow density $\boldsymbol{S}_{\omega q}(r)$ in the same way as the oscillator quantum, the electron, the electron/positron configuration and the $\mu q$, with the exception that the absorbed and re-emitted energy quanta have the frequency $\omega_{q}$. The quark $q_{1}$ can have positive and negative spin; hence, they are called $q_{1}{ }^{+}$and $q_{1}{ }^{-}$. A similar configuration of micro quarks with frequency $\omega_{\mu q 2}$ constitutes the quark type 2 which can have positive and negative spin called $q_{2}{ }^{+}$and $q_{2}{ }^{-}$. A third configuration of micro quarks with frequency $\omega_{\mu q 3}$ constitutes the quark type 3 called $q_{3}{ }^{+}$and $q_{3}{ }^{-}$. All three quarks have the same spin frequency $\omega_{q}$; however, they can have positive or negative spin.

The proton may consist of one $q_{1}{ }^{+}$, one $q_{2}{ }^{-}$and one $q_{3}{ }^{+}$. Thus, there is a total force of attraction within the proton which amalgamates the three quarks according to Equation (8). The proton also contains an embedded positron or one of the $\mu q$ contains a surplus electron. The proton has spin in itself at its natural frequency $\omega_{p}$, where $p$ denotes proton. The configuration absorbs energy quanta and re-emits energy flow density $\boldsymbol{S}_{\omega p}(r)$ in the same way as the oscillator quantum, the electron, the micro quarks and the quarks, with the exception that the absorbed and re-emitted energy quanta have the frequency $\omega_{p}$. The proton can have positive and negative spin.

The neutron may consist of one $q_{1}^{-}$, one $q_{2}{ }^{+}$and one $q_{3}{ }^{-}$. Thus there is a total force of attraction within the proton which amalgamates the three quarks according to Equation (8). The neutron also contains an embedded positron and an embedded electron or two of the $\mu q$ contain one surplus electron respectively one surplus positron. The neutron has spin in itself at its frequency $\omega_{n}$, which is the same as the protons spin frequency. The neutron can have positive or negative spin. The configuration absorbs energy quanta and re-emits energy flow density $\boldsymbol{S}_{\omega n}(r)$ in the same way as the oscillator quantum, the electron, the micro quarks and the quarks, with the exception that the absorbed and re-emitted energy quanta have the frequency $\omega_{n}$. 
The atomic nucleus consists of different configurations of protons and neutrons, depending on type of chemical element. Adjacent protons and neutrons have opposite spin which ensures force of attraction between adjacent protons and neutrons according to Equation (8). The total force of attraction decreases with larger configurations which explains why chemical elements with large atomic weight are less stable. The repelling force between positrons embedded in protons plays a part in the total force; however, smaller than the force created by $\boldsymbol{S}_{\omega n p}(r)$.

We now realize one extremely important property of the nucleus. There is a force of attraction within each layer; however, weaker forces between layers. The reason is that each layer operates at a different frequency or in different frequency domains, i.e. each layer consists of forced damped oscillators with natural frequency specific to the layer. Consequently, the interaction between layers is small resulting in a strong total force within each layer. A structure where all layers where operating on the same frequency would result in a total force close to zero. This is the enabler of the strong force or the strong forces.

The atomic nucleus has spin in itself at a frequency which is specific to the chemical element, $\omega_{c e}$, where ce denotes chemical element. The nucleus absorbs energy quanta and re-emits energy flow density $\boldsymbol{S}_{\omega c e}(r)$ in the same way as the oscillator quantum, the electron, micro quarks, quarks, protons and neutrons, with the exception that the absorbed and re-emitted energy quanta have the frequency $\omega_{c e}$. It is proposed that every electron, orbiting the nucleus, constitutes a spin in itself (i.e. the orbital constitutes the spin) and the electron has the same spin frequency, $\omega_{c e}$, as the nucleus; however, opposite spin. Consequently, all orbiting electrons are synchronized to each other. Every electron has spin in itself with frequency $\omega_{e}$ or wavelength $\lambda_{e}$. The only allowed orbitals with radius $r$ are those who satisfy $n \cdot \lambda_{e}=2 \pi r$. The force of attraction between positrons embedded in protons and orbiting electrons plays a part in the total force between nucleus and orbiting electrons together with the force created by $\boldsymbol{S}_{\omega c e}(r)$ according to Equation (8). Consequently, the electron orbital in e.g. the helium atom is different from the first orbital of e.g. the lead atom. All electron orbitals in a chemical element have the same spin frequency $\omega_{c e}$. From this follows that electrons in the outer orbitals have more kinetic energy than those in the lower orbitals.

\subsection{Gravity}

This Section explains that the force called gravity is created by a mechanism similar to the mechanism which generates forces between electrons and positrons and within the atomic nucleus; however, gravity encompasses energy quanta with frequency specific to gravity. Gravity based on the present theory has been described previously [2], although with the constraints described in Section 1. The present theoretical model provides a more stringent and simpler description of gravity. Neutrons, protons, quarks, positrons and electrons contain oscillator quanta with natural frequency $\omega_{o}$. Neutrons, protons and electrons always contain equal amount of oscillator quanta with positive spin respectively with negative spin, hence there is always the same amount of oscillator quanta with positive spin and with negative spin in bodies. Now we picture two bodies where the distance between the bodies is much larger than the distance between oscillator quanta within the bodies. There is repelling force between oscillator quanta with equal spin and force of attraction between oscillator quanta with opposite spins, according to Equation (8). These forces would normally level out resulting in a total force equal to zero. However, it is proposed that the force of attraction is slightly larger than the repelling force; this results in a small force of attraction, i.e. the gravitational force. Hence, the force $\boldsymbol{F}$ of attraction between two bodies at distance $\boldsymbol{r}$ containing $m$ respectively $n$ oscillator quanta is

$$
\boldsymbol{F}_{\text {gravity }}(r)=-\xi_{g} m n \cdot \frac{\boldsymbol{r}}{4 \pi r^{3}},
$$

where $\xi_{g}$ is the gravitational constant and the - sign denotes force of attraction. Equation (9) is derived from Equation (8).

The number of oscillator quanta $m$ in body 1 respectively $n$ in body 2 is proportional to the gravitational mass $m_{1}$ of body 1 and the gravitational mass $m_{2}$ of body 2 . We now recognize the similarity with Newton's metric law of gravity, where $G$ is Newton's gravitational constant [5]

$$
F_{\text {gravity }}(r)=\xi_{g} m n \cdot \frac{1}{4 \pi r^{2}} \approx F_{\text {Newton }}=G m_{1} m_{2} \frac{1}{r^{2}} .
$$


We also recognize the similarity with Coulomb's law $F_{\text {Coulomb }}(r)=\frac{Q_{1} Q_{2}}{4 \pi \varepsilon_{0} r^{2}}$. The reason is that the gravitational force is similar to the force between clusters of electrons, the reason being that both derive from Equation (8).

\subsection{Photon}

The nature of the photon has been described previously [2], although with the constraints described in Section 1. The process creating photons is described here in condensed form. Particles, e.g. electron, neutron and proton contain two or many oscillator quanta with natural frequency $\omega_{o}$ as described in Sections 2.4 and 2.5. Each oscillator quantum absorbs energy quanta $\delta \boldsymbol{S}_{\omega o}$ and re-emits energy quanta $\delta \boldsymbol{S}_{\omega o}$. Accelerating or decelerating an electron implies that force and energy is enforced on the electron. This energy can be described as one or many $h v$. The energy $h v$ cannot be stored in the electron and its oscillator quanta. The energy $h v$ are, as a result, superpositioned on re-emitted energy quanta $\delta \boldsymbol{S}_{\omega o}$. One energy $h v$ is superpositioned (in the oscillator quantum) on one re-emitted energy quantum $\delta \boldsymbol{S}_{\omega o}$ at a time. This new energy quantum $\delta \boldsymbol{S}_{\omega o+v}$ has the frequency $\omega_{o}$ and $v$. Radio waves, light and gamma rays are energy $h v$ super positioned on energy quanta $\delta \boldsymbol{S}_{\omega o}$. The re-emitted energy quanta can have positive and negative spin, which implies that the photon $\delta \boldsymbol{S}_{\omega o+v}$ also can have positive and negative spin. This is confirmed by the classical definition of photon spin [5] [6].

\subsection{Gravitational and Inertial Mass}

Gravitational and inertial mass have been described previously [2], although with the constraints described in Section 1 . The process behind gravitational and inertial mass is described here in condensed form.

Gravitational mass is the expression of the mechanism, i.e. atomic oscillator quanta, which absorb and re-emit energy quanta $\delta \boldsymbol{S}_{\omega o}$ and energy flow density $\boldsymbol{S} \omega_{o}(r)$. This results in gravitational force according to Equations ((9) and (10)). Consequently, the gravitational mass $m_{1}$ in Equation (10) is proportional to the number $m$ of oscillator quanta with natural frequency $\omega_{o}$ (creating gravity) in the mass or the body.

Accelerating or decelerating a mass, a particle, an electron or an atom demands enforced force and energy. The mass, particle, electron or atom cannot store this enforced energy. The enforced energy can be described as many $h v$. The enforced energy $h v$ are superpositioned on re-emitted energy quanta, comprising the energy quantum $\delta \boldsymbol{S}_{\omega o+v}$ as described in Section 2.7. Hence, enforced energy $h v$ are emitted as photons. Thus, inertial mass is the expression of the mechanism, i.e. atomic oscillator quanta, with natural frequency $\omega_{0}$, which convert enforced energy $h v$ into emitted photons $\delta \boldsymbol{S}_{\omega o+v}$. The inertial mass is proportional to the number $m$ of oscillator quanta in the mass. Consequently, the inertial mass is proportional to the gravitational mass, although inertial and gravitational mass originate from totally different processes. The process creating inertial mass is identical to the process creating photons, as described in Section 2.7. The number of oscillators in a mass or body is normally extremely large. This results in that the emitted photons have very low frequency $v$. Photons can be detected as TEM waves (Transverse Electromagnetic waves). However, TEM waves with very low frequency cannot be measured with state of the art instruments.

\section{Discussion and Conclusions}

According to the present study the atom is completely passive; forces, fields and energy are not atomic inherent properties. All properties of the atom are the result of external energy quanta which are absorbed and re-emitted by oscillator quanta and different configurations of oscillator quanta. This enables a simple description of atom, electromagnetic theory, gravity, photon and inertial mass. There is a clear distinction between energy, forces and fields on one hand and matter and particles on the other hand. Energy is mediated by energy quanta and sometimes by super positioned energy $h v$. Forces and fields are mediated by energy quanta. Matter and particles always consist of different configurations of oscillator quanta, amalgamated by absorbed and re-emitted energy quanta.

The Standard Model [1], quantum physics [12], general relativity [13], electromagnetic theory [4] [5] and string theory [14] use a different approach. There is no external influence on the atom in those sciences, except for the Higgs field [7]-[11]. Forces, fields and energy are atomic inherent properties and there is no supply of energy from the outside. For instance the electron radiates displacement (the electric field) [4] [5] which consist 
of energy; however, there is no supply of energy to the electron. It has been shown that this is theoretically impossible; it is in fact a perpetual mobile machine [3]. The atom radiates gravitons [1], which consist of energy; however, there is no supply of energy to the atom, which is a perpetual mobile machine.

State of the art physics describe forces in very different ways; the Coulomb force is mediated by an electric field [4] [5], the strong nuclear force is mediated by a particle (the gluon) [1], in the Standard Model the gravitational force is mediated by a particle (the graviton) [1] and in string theory the gravitational force is mediated by one-dimensional strings [14]. In the present study these three forces are described by the same mechanism; the interaction between energy quanta. These three forces are derived from the same equation (Equation 8); however, where each operates in its own frequency domain.

In state of the art physics the photon is an elementary particle and the force needed to accelerate a body (the inertial mass) is described by a scalar field (the Higgs Field) and a scalar boson (the Higgs boson) [7]-[9]. Attempts have also been made to extend the scalar Higgs field to incorporate other areas, e.g. gravitons [10] [11]. In the present study there is no difference between the mechanism generating photons and the mechanism resulting in inertial mass; in both cases enforced energy $h v$ is super positioned on re-emitted energy quanta and which constitutes the photon. In the present study scalar fields do not exist; the energy quantum is a vector and hence, fields are vector fields.

State of the art elementary particle physics make little distinction between energy, force and matter; all three are often described as particles. The photon, which mediates energy, is a particle [1] [6]. The gluon and the graviton, which mediate forces, are particles [1]. Matter like the electron, quarks, neutron and proton are also particles [1]. In the present study there is a clear difference between 1) energy which is mediated by energy quanta or super positioned energy $h v$ on energy quanta, 2 ) forces which are created by the interaction between energy quanta which have the same frequency and 3) matter which always consist of configurations of oscillator quanta and which are amalgamated by absorbed and re-emitted energy quanta.

In the Standard Model the quark is described by its flavors: up, down, strange, charm, top, bottom, red, blue and green [1]. In the present study only three entities are introduced: energy quanta, oscillator quantum and configurations of oscillator quanta and where all three have frequency and spin. The oscillator quantum and its configurations constitute forced damped oscillators with different natural frequencies. The definitions of energy, forced damped oscillator, frequency, natural frequency and spin are well known [4] [5].

Numerous different particles have been discovered when splitting larger particles, e.g. the neutron. It is proposed that many of these "particles" are fragments of different configurations (e.g. $\mu q$ and quarks) contained in the destroyed particle. These fragments have, initially, a spin frequency; however, the spin frequency does not equal the frequency of any energy quanta. Thus, it is unable to absorb energy quanta, resulting in that it rapidly decays. It is proposed that many observed "particles", which rapidly decay, are not particles; they are fragments which do not fulfill the criteria of absorbing and re-emitting energy quanta. They should be regarded as noise. Using these criteria will probably ease the understanding of the atom.

The future will show if the atom is extremely complex, or quite simple. If it is reasonably simple, there may exist a theory which amalgamates elementary particle physics, quantum physics, general relativity, string theory, astrophysics, electromagnetic theory and the forces within chemistry into one comprehensive theory; Theory of Everything, TOE.

\section{References}

[1] Burgess, C. and Moore, G. (2007) The Standard Model: A Primer. Cambridge University Press, Cambridge.

[2] Giertz, H.W. (2015) Journal of Modern Physics, 6, 157-167. http://dx.doi.org/10.4236/jmp.2015.62021

[3] Giertz, H.W. (2015) Journal of Basic and Applied Research International, 9, 2395-3438

[4] Bleaney, B.I. and Bleaney, B. (1965) Electricity and Magnetism. Oxford University Press, Amen House, London.

[5] Harris, R. (2008) Modern Physics. 2nd Edition, Pearson International Edition, Addison Wesley, Boston.

[6] Sipe, J.E. (1995) Physical Review A, 52, 1875-1883. http://dx.doi.org/10.1103/PhysRevA.52.1875

[7] Higgs, P.W. (1964) Physical Review Letters, 11, 508-509. http://dx.doi.org/10.1103/PhysRevLett.13.508

[8] Higgs, P.W. (1964) Physical Letters, 12, 132-133. http://dx.doi.org/10.1016/0031-9163(64)91136-9

[9] Englert, F. and Brout, R. (1964) Physical Review Letters, 13, 321-323. http://dx.doi.org/10.1103/PhysRevLett.13.321

[10] Oda, I. (2010) Physics Letters B, 690, 322-327. http://dx.doi.org/10.1016/j.physletb.2010.05.048 
[11] Bezrukov, F. and Shaposhnikov, M. (2008) Physics Letters B, 659, 703-706. http://dx.doi.org/10.1016/j.physletb.2007.11.072

[12] Eisberg, R. and Resnick, R. (1985) Quantum Physics of Atoms, Molecules, Solids, Nuclei, and Particles. 2nd Edition, Wiley, New York.

[13] Einstein, A. (1916) Annalen der Physik, 49, 285-339.

[14] Polchinski, J. (1998) String Theory. Cambridge University Press, Cambridge. 